\title{
Association between Preoperative Transthoracic Echocardiography and Clinical Outcomes after Scheduled Hip Fracture Surgery in Geriatric Patients
}

\author{
Misoon Lee, So Jeong Lee, Sang-Hyun Kim \\ Department of Anesthesiology and Pain Medicine, Soonchunhyang University Bucheon Hospital, Soonchunhyang University College of Medicine, Bucheon, \\ Korea
}

\begin{abstract}
Objective: Hip fracture is a major problem for elderly patients and is expected to become more common as society ages. Transthoracic echocardiography (TTE) is still widely performed for cardiac risk assessment, while routine echocardiography is not recommended in patients undergoing non-cardiac surgery. Thus, we evaluated the relationship between TTE-related variables and postoperative outcomes in elderly patients with hip fracture.

Methods: This study consisted of a single-center, retrospective medical record review including a total of 356 patients. The study population included patients 65 years of age and older who underwent TTE for preoperative assessment within 1 month before the scheduled hip fracture surgery between January 2013 and October 2016.

Results: In total, 356 patients were included. Seven patients died within 30 days after surgery. Advanced age (odds ratio [OR], 1.14; $95 \%$ confidence interval $[\mathrm{Cl}], 1-1.3 ; \mathrm{P}<0.05)$, increased left ventricular ejection fraction $(\mathrm{OR}, 1.26 ; 95 \% \mathrm{Cl}, 1.07-1.48 ; \mathrm{P}<0.05)$, and arrhythmia $(\mathrm{OR}, 64.99 ; 95 \% \mathrm{Cl}, 3.91-1,079.91 ; \mathrm{P}<0.05)$ were all significant risk factors for 30-day mortality. Increased left atrium volume was associated with 180-day mortality (OR, 1.02; $95 \% \mathrm{Cl}, 1-1.04 ; \mathrm{P}<0.05)$.

Conclusion: In elderly patients undergoing hip fracture surgery, preoperative TTE is not a useful predictive factor for postoperative mortality.
\end{abstract}

Keywords: Echocardiography; Hip fractures; Mortality; Geriatrics; Postoperative complications

\section{INTRODUCTION}

The incidence of hip fracture has been steadily increasing as society ages and is associated with high mortality and morbidity in the elderly population [1,2]. Hip fractures also cause enormous economic burdens. In the Republic of Korea, the total annual health care cost of treating hip fractures in people 50 years of age and older is estimated to have increased from \$137 million in 2008 to $\$ 164$ million in 2011 (i.e., by an average of $6.1 \%$ per year). The total number of hip fractures also increased by $20.8 \%$ over this 4-year period [3]. Therefore, hip fracture is considered an important medical and financial issue.

Cardiovascular issues are the most common condition in the elderly population and are a major cause of death and an independent predictive factor for mortality after noncardiac surgery [4,5].
Transthoracic echocardiography (TTE) is still widely performed to assess the risk of associated cardiovascular disease in individuals who undergo hip fracture surgery; however, based on recently published guidelines, routine echocardiography is not recommended for preoperative assessment in individuals undergoing noncardiac surgery $[6,7]$. TTE is useful for observing left ventricular wall function and is therefore used to assess cardiac risk before surgery [8], but the cost-benefit ratio of TTE remains unclear. According to several previous studies, early surgery of hip fracture (within 48 hours) reduced the length of hospital stay, perioperative complications, and mortality $[9,10]$. However, despite the recommendation of recent guidelines to avoid unnecessary echocardiography, preoperative transthoracic echocardiography for screening purposes is still being used indiscriminately. This wastes medical resources and can also delay surgery and adversely affect the prog- 
Lee M, et al. - Preoperative Echocardiography in Hip Fracture Surgery

nosis of patients. Guidelines for noncardiac surgery published in 2014 did not recommend routine TTE for perioperative cardiac risk evaluation [6]. According to this guideline, evaluation of left ventricular function is recommended for patients with dyspnea of unknown origin or with heart failure with worsening dyspnea or other changes [6]. Similarly, the guidelines for cardiovascular assessment and management of noncardiac surgery do not recommend routine echocardiography in patients undergoing intermediate-or low-risk surgery [7].

The aim of this study was to evaluate whether preoperative TTE for assessment of cardiac function had an effect on postoperative short-term mortality.

\section{MATERIALS AND METHODS}

This study consisted of a single-center, retrospective medical record review. The study population included patients 65 years of age and older who underwent TTE within a month before the scheduled surgical repair of hip fracture for preoperative cardiac risk assessment between January 2013 and October 2016 at the Department of Orthopedics in a tertiary hospital. Exclusion criteria were as follows: age younger than 65 , history of bilateral hip fracture surgery, and re-operation due to complications of previous hip surgery. The study protocol was approved by the institutional review board of Soonchunhyang University Bucheon Hospital (IRB approval no., SCHBC 2017-04-016-001). The board waived the requirement for patients' informed consent because of the retrospective design.

The primary endpoint was mortality within 30 days after surgery in patients with hip fractures who underwent TTE for assessment of preoperative cardiac risk. The secondary endpoint was the development of postoperative complications. Data included demographic characteristics such as age and gender and clinical characteristics such as height, weight, body mass index, American Society of Anesthesiologists classification, and medical history including surgery, hypertension, diabetes mellitus, coronary arterial disease, heart failure, valvular disease, arrhythmia, stroke, neurologic disease, kidney disease, and pulmonary disease. TTE-related variables included left ventricular ejection fraction (LVEF), left ventricular end-diastolic dimension, left ventricular end-systolic dimension, LVEF, ventricular mass, left ventricular mass index, mitral peak velocity of late filling, mitral peak velocity of early filling, the early diastolic mitral annular velocity of the septal mitral annulus, the ratio of mitral peak velocity of early filling to the early diastolic mitral annular velocity of the septal mitral annulus, and left atrial volume. Postoperative mortality was tracked for 180 days postoperatively. Mortality data were provided by the microdata service from Korean statistics (MDSS, mdss.kostat.go.kr). Postoperative complications were defined as newly onset delirium, car-

Table 1. Demographic characteristics, echocardiographic findings and medical histories in 356 elderly patients who underwent hip fracture surgery ( $N=356$ )

\begin{tabular}{|c|c|}
\hline Characteristic & Value \\
\hline \multicolumn{2}{|l|}{ Demographics } \\
\hline Age (yr) & $79.7 \pm 6.7$ \\
\hline \multicolumn{2}{|l|}{ Sex } \\
\hline Male & $78(21.9)$ \\
\hline Female & $278(78.1)$ \\
\hline \multicolumn{2}{|l|}{ Clinical characteristics } \\
\hline Height (cm) & $156.7 \pm 7.9$ \\
\hline Weight (kg) & $54.1 \pm 9.9$ \\
\hline Body mass index $\left(\mathrm{kg} / \mathrm{m}^{2}\right)$ & $21.9 \pm 3.8$ \\
\hline \multicolumn{2}{|c|}{ American Society of Anesthesiologists classification } \\
\hline 1 & $21(5.9)$ \\
\hline 2 & $254(71.3)$ \\
\hline$\geq 3$ & $81(22.8)$ \\
\hline \multicolumn{2}{|l|}{ Medical history } \\
\hline Hypertension & $224(62.9)$ \\
\hline Diabetes mellitus & $108(30.3)$ \\
\hline Coronary arterial disease & $37(10.4)$ \\
\hline Heart failure & $22(6.2)$ \\
\hline Valvular disease & $9(2.5)$ \\
\hline Arrhythmia & $20(5.6)$ \\
\hline Stroke & $65(18.3)$ \\
\hline Neurologic disease & $83(23.3)$ \\
\hline Kidney disease & $35(9.8)$ \\
\hline Pulmonary disease & $57(16.0)$ \\
\hline \multicolumn{2}{|l|}{ TEE-related variables } \\
\hline $\operatorname{LVEF}(\%)$ & $62.8 \pm 7.6$ \\
\hline LVEDD (mm) & $42.5 \pm 5.7$ \\
\hline LVESD (mm) & $29.1 \pm 5.3$ \\
\hline LVMI $\left(\mathrm{g} / \mathrm{m}^{2}\right)$ & $84.5 \pm 23.0$ \\
\hline LA volume (mm) & $36.6 \pm 20.8$ \\
\hline$E$ & $67.9 \pm 18.1$ \\
\hline $\mathrm{A}(\mathrm{cm} / \mathrm{sec})$ & $92.8 \pm 21.3$ \\
\hline $\mathrm{E}^{\prime}(\mathrm{cm} / \mathrm{sec})$ & $4.8 \pm 1.3$ \\
\hline $\mathrm{A}^{\prime}(\mathrm{cm} / \mathrm{sec})$ & $8.6 \pm 1.9$ \\
\hline E/E' ratio & $15.0 \pm 6.7$ \\
\hline
\end{tabular}

Values are presented as mean \pm standard deviation or number $(\%)$.

TEE, transesophageal echocardiogram; LVEF, left ventricular ejection fraction; LVEDD, left ventricular end-diastolic dimension; LVESD, left ventricular end-systolic dimension; LVMI, left ventricular mass index; $L A$, left atrium; $E$, mitral peak velocity of early filling; $A$, mitral peck velocity of late filling; $E^{\prime}$, early diastolic mitral annular velocity of the septal mitral annulus; $E / E^{\prime}$, the ration of $E$ to $E^{\prime}$. 
diovascular disease, pneumonia, urinary tract infection and renal disease, and other complications after hip fracture surgery. The definition of each complication is as follows. Delirium is diagnosed by a neurologist, cardiovascular disease is a major adverse cardiovascular event, pneumonia is diagnosed by a pulmonologist, and urinary tract infection and renal disease are diagnosed by a nephrologist.

Statistical analysis was performed using R ver. 3.3.2 (The $\mathrm{R}$ Foundation for Statistical Computing, Vienna, Austria). The significance level for all statistical tests was set at 5\% $(\mathrm{P}<0.05)$. Results are expressed as mean \pm standard deviation. Univariable and multivariable regression analyses were sequentially performed to assess the effects of TTE-related variables on 30-day mortality and the development of postoperative complications.

Table 2. Univariable regression analysis of preoperative assessment variables for mortality at 30-day

\begin{tabular}{lcc}
\hline \multirow{2}{*}{ Variable } & \multicolumn{2}{c}{$30-$ Day mortality } \\
\cline { 2 - 3 } Age $($ yr) & $1.16(1.04-1.32)$ & 0.01 \\
Female & $1.70(0.28-32.33)$ & 0.63 \\
TEE-related variables & & \\
LVEF (\%) & $1.23(1.08-1.44)$ & 0.00 \\
LVEDD (mm) & $1.05(0.90-1.21)$ & 0.50 \\
LVESD (mm) & $0.92(0.75-1.10)$ & 0.41 \\
LVMI (g/m²) & $0.99(0.95-1.03)$ & 0.61 \\
LA volume (mm) & $1.01(0.96-1.03)$ & 0.61 \\
E (cm/sec) & $1.03(0.99-1.06)$ & 0.15 \\
A (cm/sec) & $1.04(1.00-1.07)$ & 0.03 \\
E' (cm/sec) & $0.89(0.44-1.66)$ & 0.74 \\
A' (cm/sec) & $1.06(0.62-1.68)$ & 0.81 \\
E/E' ratio & $1.04(0.94-1.10)$ & 0.30 \\
Medical history & & \\
Hypertension & $0.43(0.08-2.00)$ & 0.28 \\
Diabetes mellitus & $0.92(0.13-4.33)$ & 0.92 \\
Coronary arterial disease & $1.45(0.08-8.81)$ & 0.74 \\
Heart failure & $6.58(0.90-32.70)$ & 0.03 \\
Valvular disease & $7.10(0.35-49.01)$ & 0.09 \\
Arrhythmia & $7.36(1.01-36.83)$ & 0.02 \\
Stroke & $3.47(0.67-16.13)$ & 0.11 \\
Neurologic disease & $0.54(0.03-3.24)$ & 0.57 \\
Kidney disease & $1.54(0.08-9.41)$ & 0.69 \\
Pulmonary disease & $2.14(0.30-10.20)$ & 0.37 \\
\hline
\end{tabular}

TEE, transesophageal echocardiogram; LVEF, left ventricular ejection fraction; LVEDD, left ventricular end-diastolic dimension; LVESD, left ventricular end-systolic dimension; LVMI, left ventricular mass index; LA, left atrium; E, mitral peak velocity of early filling; $A$, mitral peak velocity of late filling; $E^{\prime}$, early diastolic mitral annular velocity of the septal mitral annulus; $E / E^{\prime}$, the ration of $E$ to $E^{\prime}$.

\section{RESULTS}

\section{Patient characteristics}

Between January 2013 and October 2016, 356 patients underwent TTE for the assessment of cardiac risk before surgical repair of hip fracture. The characteristics of the patients are specified in Table 1.

\section{Clinical outcomes and analysis}

The incidence of $30,60,90$, and 180 days after surgery were $1.96 \%$, $4.21 \%, 6.18 \%$, and $8.99 \%$, respectively. Table 2 lists the odds ratios (ORs) for postoperative mortality in elderly individuals with hip fracture. Based on univariable logistic regression analysis, advanced age (OR, 1.16; 95\% confidence interval [CI], 1.04-1.32; $\mathrm{P}<0.05)$, increased LVEF (OR, 1.23; 95\% CI, 1.08-1.44; $\mathrm{P}<0.05)$ values, mitral peak velocity of late filling (OR, 1.04; 95\% CI, 1.00-1.07; P < 0.05) and arrhythmia (OR, 7.36; 95\% CI, 1.01-36.83; $\mathrm{P}<0.05)$ were associated with 30 -day mortality following surgery, respectively. Table 3 lists the ORs for the development of postoperative complications, including postoperative delirium and cardiovascular disease.

Based on multiple logistic regression analysis, advanced age (OR, 1.14; 95\% CI, 1.00-1.30; $\mathrm{P}<0.05)$, increased LVEF (OR, 1.26; 95\% CI, 1.07-1.48; $\mathrm{P}<0.05)$ and arrhythmia (OR, 64.99; 95\% CI,

Table 3. Univariable regression analysis of preoperative assessment variables for development of postoperative complications

\begin{tabular}{lcc}
\hline \multirow{2}{*}{ Variable } & \multicolumn{2}{c}{ Postoperative complication } \\
\cline { 2 - 3 } & Odds ratio (95\% confidence interval) & P-value \\
\hline Age $(\mathrm{yr})$ & $1.04(1.01-1.08)$ & 0.02 \\
Female & $0.58(0.35-0.97)$ & 0.04 \\
TEE-related variables & & \\
LVEF $(\%)$ & $1.00(0.97-1.03)$ & 0.76 \\
LVEDD $(\mathrm{mm})$ & $0.98(0.94-1.03)$ & 0.45 \\
LVESD $(\mathrm{mm})$ & $1.00(0.95-1.04)$ & 0.85 \\
LVMI $\left(\mathrm{g} / \mathrm{m}^{2}\right)$ & $1.01(0.99-1.02)$ & 0.33 \\
LA volume $(\mathrm{mm})$ & $1.01(1.00-1.02)$ & 0.13 \\
E $(\mathrm{cm} / \mathrm{sec})$ & $1.01(1.00-1.03)$ & 0.03 \\
A $(\mathrm{cm} / \mathrm{sec})$ & $1.01(1.00-1.02)$ & 0.19 \\
$E^{\prime}(\mathrm{cm} / \mathrm{sec})$ & $0.93(0.77-1.11)$ & 0.46 \\
$A^{\prime}(\mathrm{cm} / \mathrm{sec})$ & $0.96(0.84-1.09)$ & 0.54 \\
E/E' ratio & $1.03(0.99-1.06)$ & 0.16 \\
\hline
\end{tabular}

TEE, transesophageal echocardiogram; LVEF, left ventricular ejection fraction; LVEDD, left ventricular end-diastolic dimension; LVESD, left ventricular end-systolic dimension; LVMI, left ventricular mass index; LA, left atrium; $E$, mitral peak velocity of early filling; $A$, mitral peck velocity of late filling; $E^{\prime}$, early diastolic mitral annular velocity of the septal mitral annulus; $E / E^{\prime}$, the ration of $E$ to $E^{\prime}$. 
Lee M, et al. - Preoperative Echocardiography in Hip Fracture Surgery

3.91-1,079.91; $\mathrm{P}<0.05)$ were all significant risk factors for 30-day mortality. Increased left atrial volume was a risk factor for 180-day mortality (OR, 1.02; 95\% CI, 1-1.04; $\mathrm{P}<0.05)$. Coronary arterial disease (OR, 2.73; 95\% CI, 1.19-6.26; $\mathrm{P}<0.05)$ and neurologic disease (OR, 2.88; 95\% CI, 1.54-5.36; $\mathrm{P}<0.05)$ were significant risk factors for the development of postoperative complications.

\section{DISCUSSION}

This study investigated the utility of preoperative thoracic echocardiography in predicting preoperative mortality in elderly patients undergoing hip fracture surgery. We found that increased LVEF was a risk factor for 30-day mortality, which contradicted the results of several previous studies. This result requires attention to interpretation. We first considered that this study was limited to elderly patients over 65 years as the cause of these findings. The aging process is closely related to changes in cardiac structure [11-13]. Morphological and structural changes in the heart with increasing age are common findings. The cardiomyocyte dies with aging, leading to hypertrophy of the remaining cells due to the counterbalance mechanism for the reduced number of cells [13]. Due to this mechanism, several studies have been published showing the results of the preservation of systolic function in the elderly population as opposed to a decrease in diastolic ventricular function $[14,15]$. In addition, in a previous study using cardiac magnetic resonance, the mean value of LVEF was highest in the group over 70 years old, which is also suitable for interpreting as a structural change in the heart due to the aging process [16]. Another possible cause is that the patients included in the present study have heart failure with preserved left ventricular systolic function that has not been diagnosed. The prevalence of preserved systolic function is between $40 \%$ and $71 \%$, and about half of heart failure patients have a normal range of LVEF values at echocardiography [17]. According to a retrospective study published in 2010, severely reduced LVEF (<30\%) was an independent predictive factor for adverse perioperative outcomes and long-term mortality in elderly patients who underwent intermediate and highrisk noncardiac surgery [18]. This result suggested that preoperative echocardiography is not a useful independent predictor of 30day mortality. In contrast, increased left atrial volume had an effect on 180-day mortality. It is clinically important to measure left atrial volume during echocardiography to assess diastolic function and left ventricular filling pressure [19]. Enlarged left atrium is as- sociated with a number of severe adverse events, such as atrial fibrillation, valvular disease, stroke, heart failure, and cardiovascular death. Age-related diastolic dysfunction is a common phenomenon. In addition, of the TTE-related variables, mitral peak velocity of late filling was found to be associated with 30-day mortality, which also seems to be related to cardiac diastolic disorders. Thus, the association between left ventricular volume and 180-day mortality may be the cause of impaired diastolic function due to the aging process.

In 2012, Canty et al. [20] reported the utility of the assessment of preoperative cardiac risk using echocardiography in elderly individuals scheduled for surgical repair of hip fracture. They found that focused TTE performed by trained anesthesiologists was associated with lower mortality in patients at increased cardiac risk compared with patients who did not undergo echocardiography before surgery [6]. Nevertheless, the clinical benefit of preoperative TTE in asymptomatic patients undergoing noncardiac surgery remains unclear [21]. Echocardiography is undoubtedly a useful diagnostic technique for assessing left ventricular function in patients at increased risk for cardiac complications [22], but routine echocardiography is not recommended in patients undergoing low-or intermediate-risk surgery [6,7]. In 2015, Luttrell and Nana [21] demonstrated that preoperative TTE is not associated with lower mortality in elderly patients with hip fracture.

Furthermore, preoperative TTE can delay surgery. A previous study found that TTE significantly delayed surgical repair in hip fracture patients by an average of 31.7 hours, with $52 \%$ of patients delayed for more than 48 hours [21]. Such delays not only increase medical costs but also affect postoperative outcomes. According to several published studies, early surgery within 48 hours of admission is recommended $[9,10,23]$. Early surgical repair of hip fracture has been shown to reduce postoperative mortality and morbidity, improve function, and shorten the length of hospital stay. Furthermore, early surgery is not associated with adverse outcomes.

In addition to TTE-related variables, pre-existing arrhythmias were found to affect 30-day mortality. This finding is consistent with previous reports that arrhythmia affects perioperative mortality and length of hospital stay [24]. Age was also found to affect short-term mortality [21,25-27]. According to a previous study by Dodd et al. [28], the incidence of 30-day mortality is significantly higher in older patients, and several previous studies have also reported that advanced age affects postoperative mortality [29]. 
It is important to note several limitations of the present study. First, this study employed a retrospective medical record review from a single center. Data collected from more than 300 subjects were analyzed based on the medical records accumulated over a period of 3 years at a tertiary medical institution. We did not directly obtain adverse outcomes, including postoperative complications and mortality. To overcome these limitations, we requested microdata services from Korean statistics (MDSS, mdss.kostat. go.kr) for 180 days after hip fracture surgery, and used this microdata to accurately assess postoperative mortality. This use of microdata was a strength of this study and enhanced the reliability of the findings. Using these data from the Korean government, we were able to assess 30 -day mortality without using telephone calls or postal mail. However, no data were available regarding the precise cause of death, so more multi-center, prospective research is needed to address the limitations of the present study.

In conclusion, preoperative TTE may not only improve the postoperative outcome but may also delay surgical treatment in elderly patients who underwent scheduled for hip fracture surgery.

\section{REFERENCES}

1. Parker M, Johansen A. Hip fracture. BMJ 2006;333:27-30.

2. Chesser T, Chauhan G, Kelly M. Management of hip fractures in the elderly. Surgery (Oxford) 2016;34:440-3.

3. Kim HY, Ha YC, Kim TY, Cho H, Lee YK, Baek JY, et al. Healthcare costs of osteoporotic fracture in Korea: information from the National Health Insurance claims database, 2008-2011. J Bone Metab 2017;24:125-33.

4. Van Waes JA, Nathoe HM, de Graaff JC, Kemperman H, de Borst GJ, Peelen LM, et al. Myocardial injury after noncardiac surgery and its association with short-term mortality. Circulation 2013;127:2264-71.

5. Landesberg G, Beattie WS, Mosseri M, Jaffe AS, Alpert JS. Perioperative myocardial infarction. Circulation 2009;119:2936-44.

6. Fleisher LA, Fleischmann KE, Auerbach AD, Barnason SA, Beckman JA, Bozkurt B, et al. 2014 ACC/AHA guideline on perioperative cardiovascular evaluation and management of patients undergoing noncardiac surgery: executive summary: a report of the American College of Cardiology/American Heart Association Task Force on Practice Guidelines. Circulation 2014;130:2215-45.

7. Kristensen SD, Knuuti J, Saraste A, Anker S, Botker HE, Hert SD, et al. 2014 ESC/ESA guidelines on non-cardiac surgery: cardiovascular assessment and management: the Joint Task Force on non-cardiac surgery: cardiovascular assessment and management of the European Society of Cardiology (ESC) and the European Society of Anaesthesiology (ESA). Eur Heart J 2014;35:2383-431.

8. Halm EA, Browner WS, Tubau JF, Tateo IM, Mangano DT. Echocardiography for assessing cardiac risk in patients having noncardiac surgery: study of Perioperative Ischemia Research Group. Ann Intern Med 1996; 125:433-41.

9. Khan SK, Kalra S, Khanna A, Thiruvengada MM, Parker MJ. Timing of surgery for hip fractures: a systematic review of 52 published studies in- volving 291,413 patients. Injury 2009;40:692-7.

10. Orosz GM, Magaziner J, Hannan EL, Morrison RS, Koval K, Gilbert M, et al. Association of timing of surgery for hip fracture and patient outcomes. JAMA 2004;291:1738-43.

11. Gerstenblith G, Frederiksen J, Yin FC, Fortuin NJ, Lakatta EG, Weisfeldt ML. Echocardiographic assessment of a normal adult aging population. Circulation 1977;56:273-8.

12. Kaku K, Takeuchi M, Otani K, Sugeng L, Nakai H, Haruki N, et al. Ageand gender-dependency of left ventricular geometry assessed with realtime three-dimensional transthoracic echocardiography. J Am Soc Echocardiogr 2011;24:541-7.

13. Sheydina A, Riordon DR, Boheler KR. Molecular mechanisms of cardiomyocyte aging. Clin Sci (Lond) 2011;121:315-29.

14. Ruan Q, Nagueh SF. Effect of age on left ventricular systolic function in humans: a study of systolic isovolumic acceleration rate. Exp Physiol 2005; 90:527-34.

15. Nikitin NP, Loh PH, de Silva R, Witte KK, Lukaschuk EI, Parker A, et al. Left ventricular morphology, global and longitudinal function in normal older individuals: a cardiac magnetic resonance study. Int J Cardiol 2006; 108:76-83.

16. Fiechter M, Fuchs TA, Gebhard C, Stehli J, Klaeser B, Stahli BE, et al. Agerelated normal structural and functional ventricular values in cardiac function assessed by magnetic resonance. BMC Med Imaging 2013;13:6.

17. Hogg K, Swedberg K, McMurray J. Heart failure with preserved left ventricular systolic function; epidemiology, clinical characteristics, and prognosis. J Am Coll Cardiol 2004;43:317-27.

18. Healy KO, Waksmonski CA, Altman RK, Stetson PD, Reyentovich A, Maurer MS. Perioperative outcome and long-term mortality for heart failure patients undergoing intermediate- and high-risk noncardiac surgery: impact of left ventricular ejection fraction. Congest Heart Fail 2010; 16:45-9.

19. Nagueh SF, Appleton CP, Gillebert TC, Marino PN, Oh JK, Smiseth OA, et al. Recommendations for the evaluation of left ventricular diastolic function by echocardiography. Eur J Echocardiogr 2009;10:165-93.

20. Canty DJ, Royse CF, Kilpatrick D, Williams DL, Royse AG. The impact of pre-operative focused transthoracic echocardiography in emergency non-cardiac surgery patients with known or risk of cardiac disease. Anaesthesia 2012;67:714-20.

21. Luttrell K, Nana A. Effect of preoperative transthoracic echocardiogram on mortality and surgical timing in elderly adults with hip fracture. J Am Geriatr Soc 2015;63:2505-9.

22. Rohde LE, Polanczyk CA, Goldman L, Cook EF, Lee RT, Lee TH. Usefulness of transthoracic echocardiography as a tool for risk stratification of patients undergoing major noncardiac surgery. Am J Cardiol 2001;87: 505-9.

23. Khan SK, Shirley MD, Glennie C, Fearon PV, Deehan DJ. Achieving best practice tariff may not reflect improved survival after hip fracture treatment. Clin Interv Aging 2014;9:2097-102.

24. Polanczyk CA, Goldman L, Marcantonio ER, Orav EJ, Lee TH. Supraventricular arrhythmia in patients having noncardiac surgery: clinical correlates and effect on length of stay. Ann Intern Med 1998;129:279-85.

25. Forni S, Pieralli F, Sergi A, Lorini C, Bonaccorsi G, Vannucci A. Mortality after hip fracture in the elderly: the role of a multidisciplinary approach and time to surgery in a retrospective observational study on 23,973 patients. Arch Gerontol Geriatr 2016;66:13-7.

26. Sanz-Reig J, Salvador Marin J, Ferrandez Martinez J, Orozco Beltran D, Martinez Lopez JF, Quesada Rico JA. Prognostic factors and predictive model for in-hospital mortality following hip fractures in the elderly. Chin J Traumatol 2018;21:163-9. 
27. Mitchell R, Harvey L, Brodaty H, Draper B, Close J. One-year mortality after hip fracture in older individuals: the effects of delirium and dementia. Arch Gerontol Geriatr 2017;72:135-41.

28. Dodd AC, Bulka C, Jahangir A, Mir HR, Obremskey WT, Sethi MK. Predictors of 30-day mortality following hip/pelvis fractures. Orthop Trau- matol Surg Res 2016;102:707-10.

29. Canty DJ, Royse CF, Kilpatrick D, Bowyer A, Royse AG. The impact on cardiac diagnosis and mortality of focused transthoracic echocardiography in hip fracture surgery patients with increased risk of cardiac disease: a retrospective cohort study. Anaesthesia 2012;67:1202-9. 